\title{
The urea cycle is transcriptionally controlled by hypoxia-inducible factors
}

Charandeep Singh ${ }^{1}$, Andrew Benos ${ }^{1}$, Allison Grenell1,2, Vincent Tran ${ }^{1}$, Demiana Hanna $^{1}$, Bela Anand-Apte ${ }^{1,3}$, Henri Brunengraber ${ }^{4}$, Jonathan E. Sears ${ }^{1,5}$

${ }^{1}$ Ophthalmic Research, Cole Eye Institute, Cleveland Clinic, Cleveland, $\mathrm{OH} 44195$, USA.

${ }^{2}$ Department of Pharmacology, Case Western Reserve University School of Medicine, Cleveland, $\mathrm{OH}$ 44106, USA.

${ }^{3}$ Department of Ophthalmology and Department of Molecular Medicine, Cleveland Clinic Lerner College of Medicine at Case Western Reserve University, Cleveland, $\mathrm{OH}$ 44195, USA.

${ }^{4}$ Department of Nutrition, Case Western Reserve School of Medicine Cleveland, Cleveland, $\mathrm{OH}, 44106$, USA.

${ }^{5}$ Cardiovascular and Metabolic Sciences, Cleveland Clinic, Cleveland, OH 44195, USA.

\section{Keywords}

Transcriptional regulation, stable-isotope, carbamoyl phosphate synthetase 1, arginase 1, FG-4592, urea cycle, HIF

\section{Abbreviations}

HIF PHi, hypoxia inducible factor-prolyl hydroxylase inhibition; OIR, oxygen induced retinopathy; CPS1, Carbamoyl phosphate synthetase I; ARG1, Arginase 1; ARG2, Arginase 2; OTC, Ornithine transcarbamylase; HNF3- $\beta$, Hepatocyte nuclear factors; 


\begin{abstract}
Here, we demonstrate transcriptional regulation of urea cycle genes CPS1 and ARG1 by hypoxia-inducible factors (HIFs) and demonstrate a hepatic HIF dependent increase in urea cycle activity.
\end{abstract}

\title{
Introduction
}

The urea cycle is a liver specific pathway responsible for the removal of ammonia in ureotelic and uricotelic animals. Recently, recycling of ammonia has been shown to accelerate the proliferation rate of cancer cells $(1,2)$. Molecular nitrogen is not directly used by enzymes in the human body; we rather acquire our nitrogen from ammonia fixed into amino acids and proteins by microbes and legumes. Therefore, to preserve nitrogen for biosynthetic needs, the metabolism of nitrogenous compounds is very tightly regulated.

Conversion of ammonia to urea or orotate in mammals takes place in the liver, where it is first converted to carbamoyl phosphate. Enzymatic conversion of ammonia into carbamoyl phosphate, which is synthesized by Carbamoyl Phosphate Synthetase 1 (CPS1), is a rate-limiting step of the urea cycle. Carbamoyl phosphate has two fates, either to enter the urea cycle or to produce orotate-which is necessary for nucleotide synthesis and to transfer electrons to newly discovered electron transport chain component $\mathrm{DHODH}(3)$. If carbamoyl phosphate enters the urea cycle, arginine is synthesized from ammonia, which is converted either to urea or nitric oxide. The enzyme Arginase 1 (ARG1), a rate limiting enzyme in ureagenesis, in the liver converts arginine into urea and ornithine. The liver specific urea cycle is depicted in Figure 1. In addition to urea cycle, Arginase 2 (ARG2) activity is a rate limiting step in the polyamine synthesis and controls proliferation in some cell types (4). The liver specific urea cycle is depicted in Figure 1.

Glucagon, glucocorticoids and insulin are known hormonal regulators of most urea cycle enzymes (5). Glucagon and glucocorticoids have transcriptional control of ASS1, ASL, and ARG1, stabilization of CPS1 mRNA and ARG1, and the protein stabilization of ornithine transcarbamylase (OTC) $(6,7)$. Additionally, transcriptional activators that control CPS1 expression are C/EBP and Hepatocyte Nuclear Factor 3 (HNF3- $\beta$ ) (5, 8, 9). The only known repressor of urea cycle genes CPS1, OTC and ARG1 transcriptionally is p53 (10).

\section{Results and Discussion}

We previously demonstrated that a HIF prolyl hydroxylase inhibitor (HIF PHi)/stabilizer of HIF upregulated urea cycle metabolites in hyperoxic mice (11). To establish a direct 
connection of HIFs to urea cycle enzymes, we did qPCR analyses of a few urea cycle genes in primary hepatocytes isolated from 12 week old. We found increased expression of CPS1 when cells were treated with FG-4592, a HIF PHi (Fig. 2a). We also found increased expression of ARG1 when cells were treated with FG-4592 (Fig. 2b).

We next tested to see if siRNA based knockdown (KD) of HIF1 $\alpha$ and EPAS1 could modulate the expression levels of CPS1 and ARG1. Expression levels of CPS1 and ARG1 were decreased in the HIF1 $\alpha$ and EPAS1 KD conditions, however, there were still some remaining transcripts in single KDs. We found a synergistic effect of HIF1 and EPAS1 KD on transcriptional activity of these genes. In the double KD, expression of CPS1 and ARG1 was near negligible (Figs. 2c and d). We measured the expression levels of HIF1 $\alpha$ to ascertain that the synergistic effect was not due to cross- reactivity of HIF1 $\alpha$ and EPAS1 siRNAs. We rather saw normal levels of HIF1 $\alpha$ in the EPAS1 KD cells and near normal levels of EPAS1 in HIF1a KD (Figs. 2e and f).

To further confirm our findings at metabolic levels in vivo, we injected $\left[{ }^{15} \mathrm{~N}_{1}\right]$ ammonium chloride intraperitoneally in P12 mice that were treated with FG-4592 6h before ammonium chloride injections to stabilize HIF before measuring incorporation of labeled nitrogen. After injecting the $\left[{ }^{15} \mathrm{~N}_{1}\right]$ labeled ammonium chloride, livers from mice were dissected at 5, 8 and 15 minutes. FG-4592 injected animals had higher incorporation of labeled nitrogen from $\left[{ }^{15} \mathrm{~N}_{1}\right.$ ] ammonium into $\mathrm{M} 1$ labeled urea in the treated group versus in the controls (Figs. $3 a$, b and d). Liver Citrulline labeling was measured following $\left[{ }^{15} N_{1}\right]$ ammonium chloride injections and found to be higher in the FG-4592 treated animals (Fig. 3c). Plasma Arginine and Citrulline labeling were also investigated, and the maximal activity, measured as maximal initial enzyme velocity in the presence of excess substrate, in FG-4592 treated animals was higher than in control animals (Fig. 3e and f) indicating higher flux through the urea cycle.

Known and unknown regulators of the urea cycle are depicted in Figure 4. Regulation by hormones occurs through the modulation of transcriptional regulation, mRNA stability, and protein stability (6). On the protein levels, post translational modification of Sirt3 and Sirt5 have been linked to deacetylation of urea cycle enzymes - thereby upregulating urea cycle activity $(12,13)$. . Li et. al. reported that p53 repressed expression of urea cycle genes CPS1, OTC and ARG1 (10) and also found increased expression of urea cycle genes in colon cancer cells, which implies a connection between hypoxia and urea cycle activity (10). Others and we have linked the urea cycle to the mouse model of oxygen induced retinopathy, which has alternating environments of hyperoxia and hypoxia $(11,14)$. Here, we definitively demonstrate that both mitochondrial CPS1 and cytosolic Arg1 are upregulated by HIF, providing global regulation of the urea cycle. We speculate that there are two ways HIFs can activate urea cycle gene expression: either by directly binding to hypoxia response elements on these genes or by repression of p53 activity which indirectly will activate expression of urea cycle genes. 


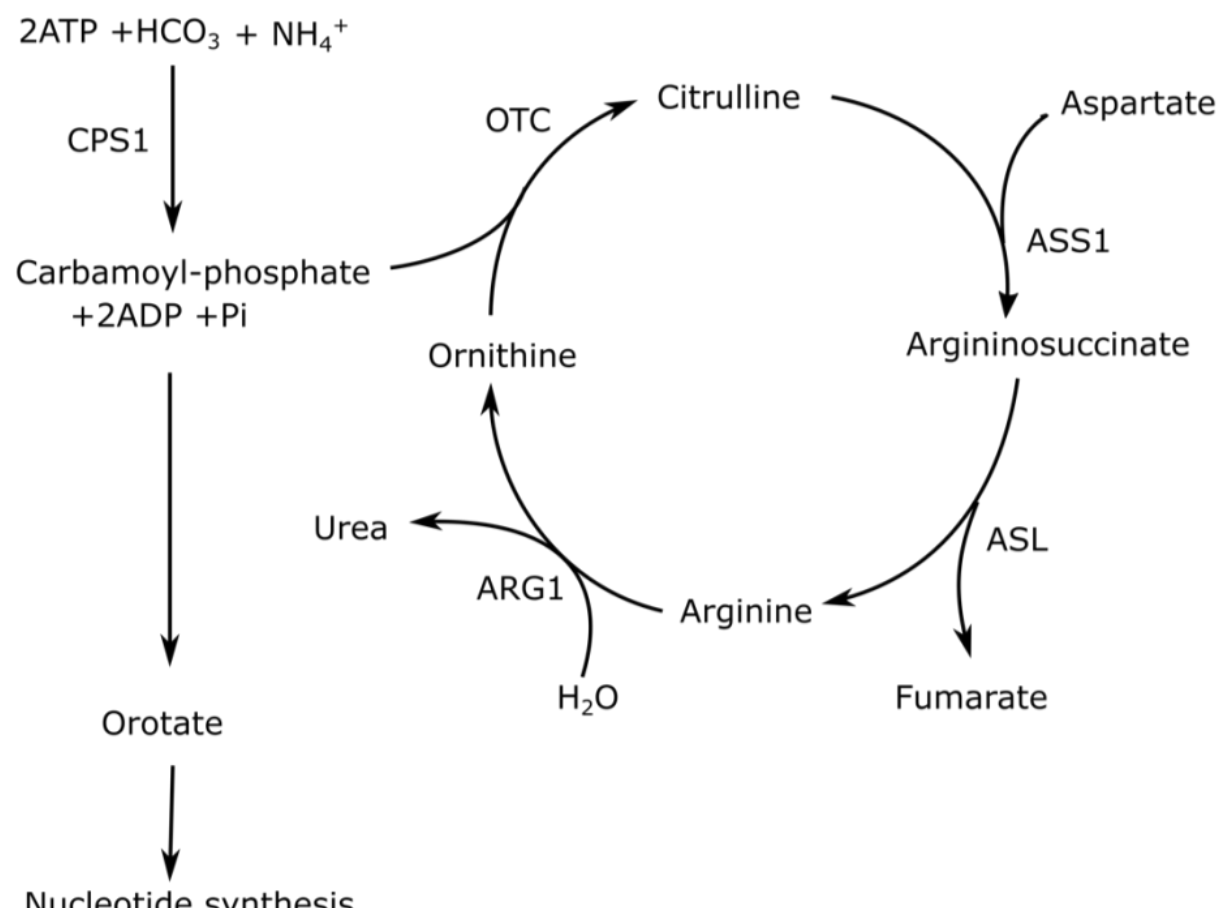

Figure 1. Urea cycle. Depicted are the liver specific urea cycle pathway enzymes and metabolites. Earlier studies have highlighted that the first enzyme of the pathway, CPS1, is only present in the liver or primary hepatocytes. CPS1 is the rate liming enzyme in the urea cycle pathway and it controls production of not just urea but also precursors for nucleotide synthesis and substrate for newly discovered electron transport chain component DHODH. Out of all the enzymes depicted in the picture, only CPS1 and ARG1 are rate limiting for urea cycle activity, with former playing roles more than merely in urea production and later controlling flux distribution between nitric oxide vs. ureagenesis. 
a)

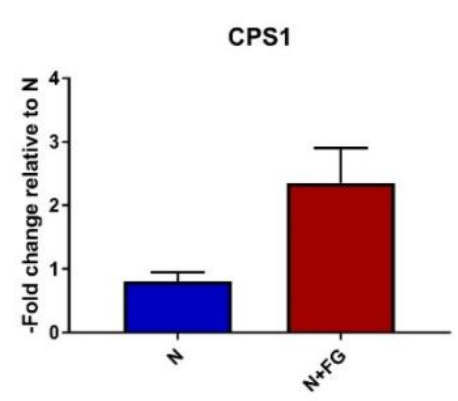

c)

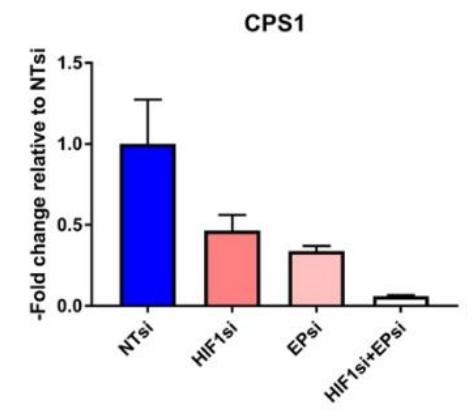

e)

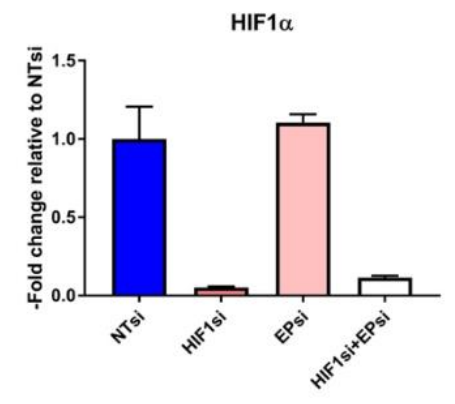

b)

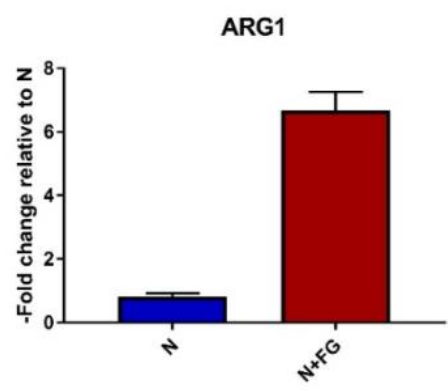

d)

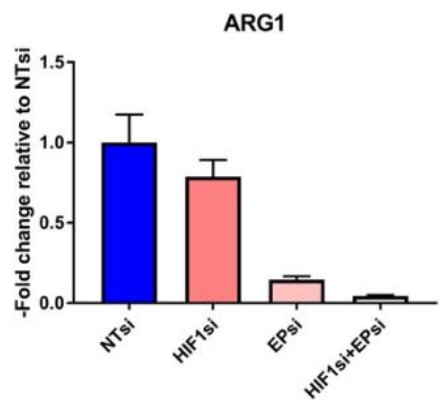

f)

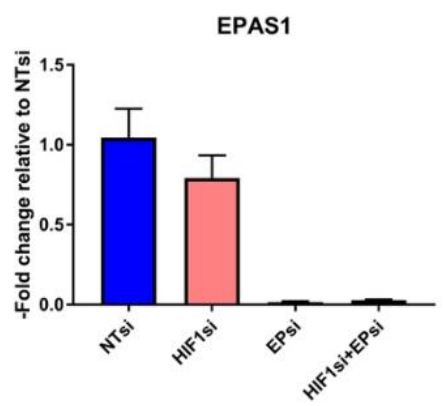

Figure 2 HIF controls expression of CPS1 and ARG1. Expression levels of a) CPS1 and b) ARG1 were found to be increased in response to FG-4592 treatment. Expression levels of c) CPS1 and d) ARG1 were downregulated in hepatocytes treated with siRNA against HIF1a or EPAS1. We measured expression of e) HIF1 $\alpha$ and f) EPAS1 in hepatocytes treated with siRNAs against HIF1 $\alpha$ and EPAS1 to confirm these siRNAs were specific for each HIF isomer. Primary hepatocytes were cultured in normoxia or hyperoxia for $24 \mathrm{~h}$ with or without FG-4592 treatment. Gene expression fold change was calculated using $2^{-\Delta \Delta C t}$ method. Gene expression was normalized to $\beta$-actin (ACTB) which served as the housekeeping control. Conditions: $N$, Normoxia; N + FG, Normoxia + FG-4592 treated. Legends: NTsi, non-targeting siRNA; HIF1si, HIF1a siRNA; EPsi, EPAS1 siRNA; HIF1si+EPsi, HIF1a siRNA+ EPAS1 siRNA. 
a)

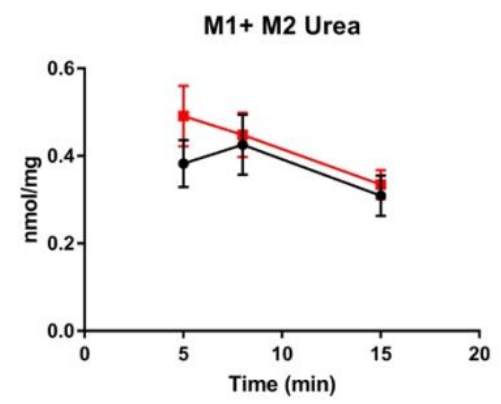

c)

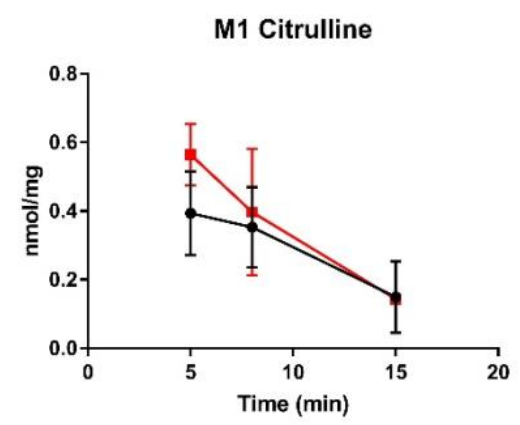

e)

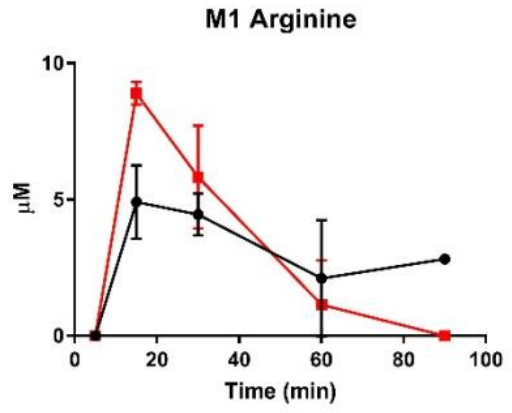

b)

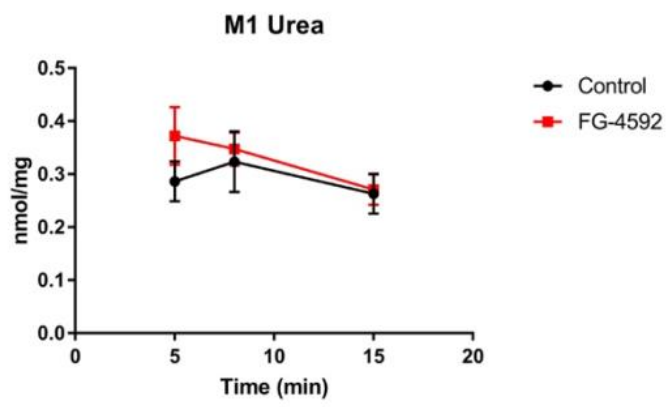

d)

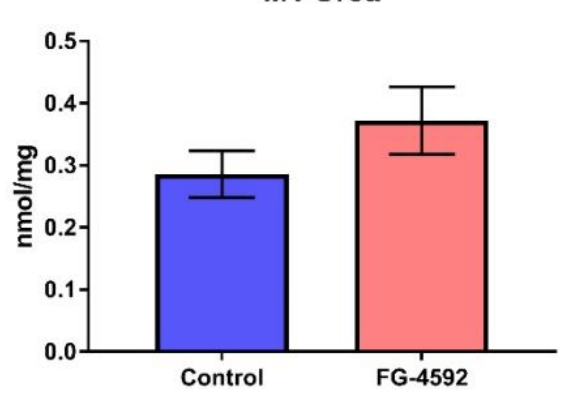

f)

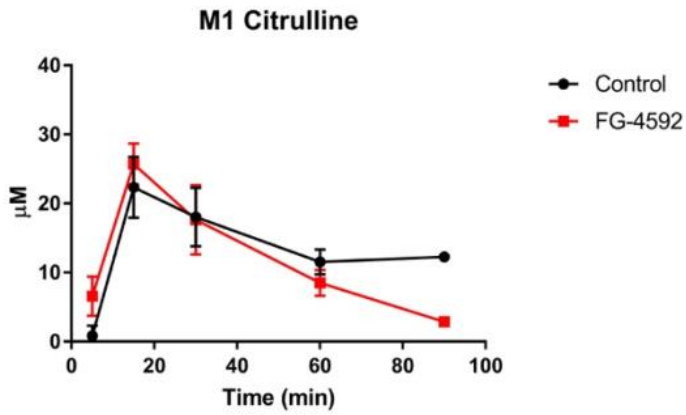

Figure 3. HIF stabilization by FG-4592 increases urea cycle flux in vivo. The absolute rates of liver specific (a) M1+M2 urea, (b) M1 urea, (c) M1 citrulline, and (d) histogram representation of M1 Urea at $5 \mathrm{~min}$. Error bars represent standard deviations. $(n=3,5 \mathrm{~min}$ control; $n=4,8 \mathrm{~min}$ control; $n=3$, 15 min control; $n=4,5$ min FG-4592; $n=4,8$ min FG-4592; $n=3,15$ min FG-4592). e) M1 Arginine time course for mouse plasma following injection of $\left[{ }^{15} \mathrm{~N}_{1}\right]$ ammonium chloride tracer ( $n=3$ for $5 \mathrm{~min}, 15 \mathrm{~min}$, and $30 \mathrm{~min} ; \mathrm{n}=2$ for $60 \mathrm{~min}$ and $90 \mathrm{~min}$ ) with and without systemic HIF stabilization. f) M1 Citrulline time course for mouse plasma following injection of $\left[{ }^{15} \mathrm{~N}_{1}\right]$ ammonium chloride tracer with and without (Figs $3 e-f, n=3$ for $5 \min , 15 \min$, and $30 \mathrm{~min} ; \mathrm{n}=2$ for $60 \mathrm{~min}$ and $90 \mathrm{~min}$ ). 


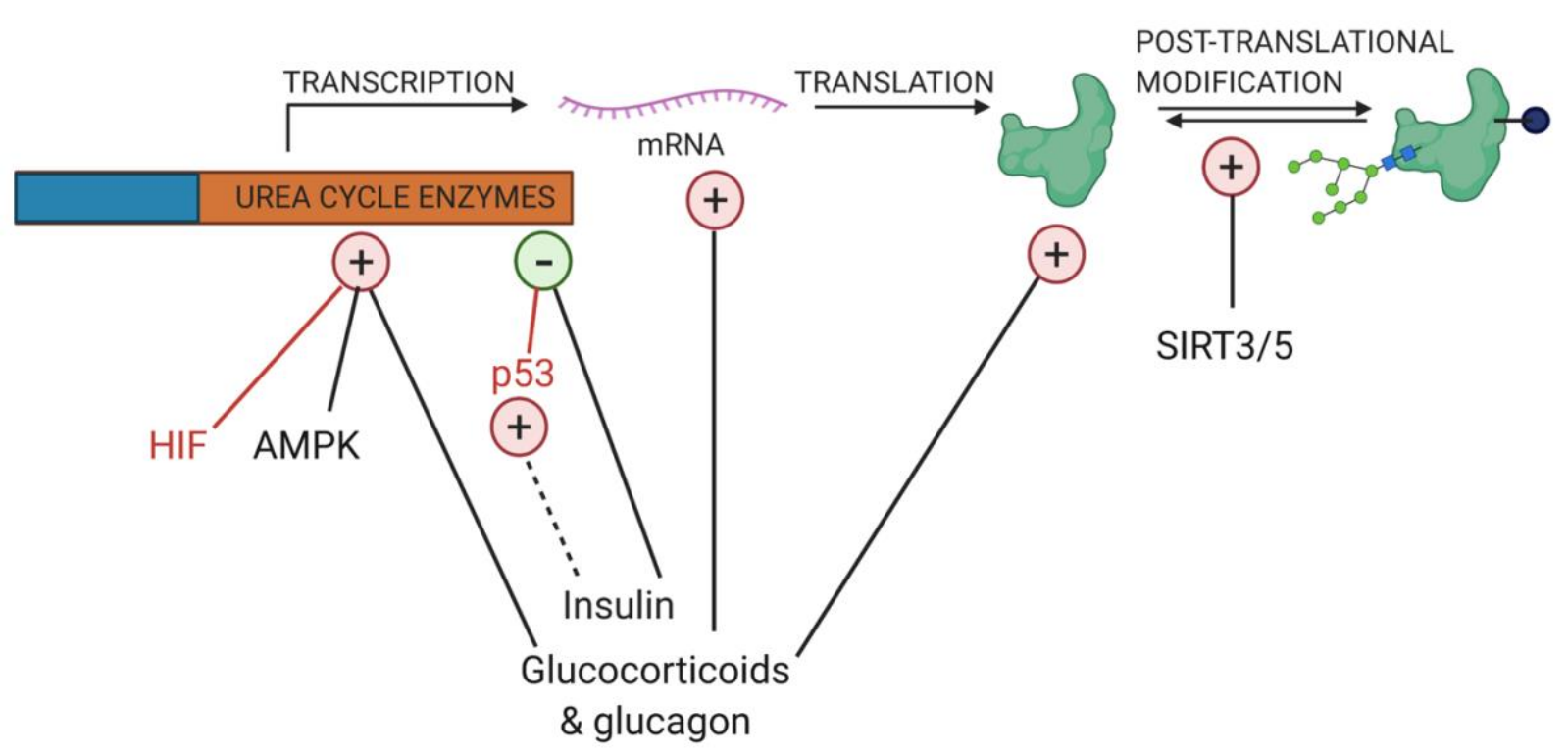

Figure 4 Known and unknown regulators of the urea cycle. Glucagon and glucocorticoids upregulate activity of urea cycle, whereas, insulin downregulates urea cycle activity. Sirt3/5 upregulates urea cycle activity by posttranslational modification of only mitochondrial urea cycle enzymes. 


\section{Methods}

\section{$\left[{ }^{15} \mathrm{~N}_{1}\right]$ ammonium chloride labeling of urea cycle compounds in vivo (liver)}

Animals were kept in normal room air until p12. On p-12, pups were given $10 \mathrm{mg} / \mathrm{Kg}$ of FG-4592 subcutaneously and then around $6 \mathrm{~h}$ post injection $50 \mu \mathrm{l}$ of $2.5 \mathrm{mg} / \mathrm{ml}$ of [ ${ }^{15} \mathrm{~N}_{1}$ ] ammonium chloride intraperitoneally $(0.5 \mu \mathrm{mol} / \mathrm{gm}$ of body weight). Following the injection of ammonium chloride tracer, mice were anesthetized with isoflurane and then sacrificed at different time points. Livers were isolated and frozen on dry ice immediately.

\section{$\left[{ }^{15} \mathrm{~N}_{1}\right]$ ammonium chloride labeling of urea cycle compounds in vivo (plasma)}

Animals were kept in normal room air until p10. On p-10, pups were given $10 \mathrm{mg} / \mathrm{Kg}$ of FG-4592 intraperitoneally and then around $4 \mathrm{~h}$ post injection $50 \mu \mathrm{l}$ of $10 \mathrm{mg} / \mathrm{ml}$ of ammonium chloride subcutaneously. Post injection of ammonium chloride tracer, mice were sacrificed at different time points, $5 \mathrm{~min}, 15 \mathrm{~min}, 30 \mathrm{~min}, 60 \mathrm{~min}$, and $90 \mathrm{~min}$. Blood was isolated from heart and added to heparin containing tubes and then centrifuged at $1020 \mathrm{x} \mathrm{g}$ for $20 \mathrm{~min}$ at $4^{\circ} \mathrm{C}$.

Note: The doses of ammonium chloride were chosen close what has earlier been used in human subjects $(15,16)$. We recommend using lower dose if infusion experiment is deemed necessary, for example as used in Yang et. al.(17).

\section{Labeling data analysis}

Areas of all mass isotopomers were extracted using masshunter data analysis software (Agilent). Data were corrected for naturally occurring istopes using Isocor software. Simultaneously, total quantities were calculated first by obtaining ratios of total urea/internal standard and then quantified using external calibrations curve of urea/internal standard at different ranges. Following formula was used to calculate the quantities of each isotopmer

Total quantity ( $\mu \mathrm{mol} / \mathrm{mg}$ of tissue) $\mathrm{x}$ isocor corrected fraction of M1 or M2 out of total value of $1=$ Quantities of M1 or M2 labeled compounds in $\mu \mathrm{mol} / \mathrm{mg}$ of tissue

\section{Metabolite extraction from the livers}

An extraction buffer consisting of $40 \mathrm{ml}$ of $80 \%$ methanol and $400 \mu \mathrm{L}$ of $\left[{ }^{13} \mathrm{C}_{5}\right]$ ribitol $(0.05 \mathrm{mg} / \mathrm{ml})$ was prepared and kept at $-20^{\circ} \mathrm{C}$ for use. Liver samples were removed from $-80^{\circ} \mathrm{C}$ and thawed on ice. These samples were weighed, transferred into fresh tubes, and kept on ice. $250 \mu \mathrm{l}$ of cold extraction buffer was added to each tube. The liver samples were homogenized using fresh disposable pestles then sonicated. The homogenized samples were then stored in $-80^{\circ} \mathrm{C}$ freezer for 20 min to quench the metabolism. The samples were then removed and transferred into fresh tubes containing additional cold extraction buffer so that the final concentration of tissue in each sample was $25 \mu \mathrm{l}$ (buffer)/mg (tissue). The samples were then vortexed and centrifuged at $3,000 \times \mathrm{g}$ for $10 \mathrm{~min}$ at $\leq 4^{\circ} \mathrm{C}$. $500 \mu \mathrm{L}$ of supernatant was transferred into 
fresh tubes and dried over night at $-4^{\circ} \mathrm{C}$ in a refrigerated centrivap concentrator (Labconco). The dried samples were then derivatized for GCMS measurement. All the samples were measured on EI-GCMS.

\section{Metabolite extraction from the plasma samples}

Five microliters of plasma sample was added to $160 \mu \mathrm{l}$ of $-20^{\circ} \mathrm{C}$ cold acetonitrile and additional $35 \mu$ of water was added to the samples. Samples were vortexed and immediately centrifuged at $15000 \times \mathrm{g}$ for $5 \mathrm{~min}$ at $4^{\circ} \mathrm{C}$. Supernatant $150 \mu \mathrm{l}$ was dried with 10 ul of $0.05 \mathrm{mg} / \mathrm{ml}$ of $\left[{ }^{13} \mathrm{C}_{5}\right]$ ribitol, overnight at $-4^{\circ} \mathrm{C}$ in a refrigerated centrivap concentrator (Labconco).

\section{Derivatization of samples for GCMS measurement}

Dried samples were removed from the centrivap concentrator (Labconco). $25 \mu \mathrm{L}$ of Methoxyamine hydrochloride/pyridine (sigma Aldrich) solution (40 mg/ml) were added to each tube. The samples were mixed at $45^{\circ} \mathrm{C}$ at $1,000 \mathrm{rpm}$ for $30 \mathrm{~min}$ on the ThermoMixer (Eppendorf). $75 \mu \mathrm{L}$ of MSTFA + 1\% TMCS (Thermo Scientific) were added to each tube and the tubes were mixed at $45^{\circ} \mathrm{C}$ at $1,000 \mathrm{rpm}$ for a second 30minute period. The samples were removed from the ThermoMixer and were allowed to cool to room temperature before centrifugation at $15,000 \times \mathrm{g}$ for $3 \mathrm{~min}$ (room temperature). Approximately $60 \mu \mathrm{l}$ of supernatant were packed into GCMS vials, out of which 1ul was injected. GCMS method use for measurement was same as described earlier in Singh et. al. (2020) with slight changes: column used here was DB-5MSDG $40 \mathrm{~m} \times 250 \mu \mathrm{m} \times 0.25 \mu \mathrm{m}$ (Agilent) and post-column re-equilibration step was removed (18).

\section{Cell culture, RNA extraction and cDNA preparation}

Cells were seeded in 6-well plates in Williams $\mathrm{E}$ media at a staring density of $0.3 \times 10^{6}$ cells per well. Plates were incubated in $5 \% \mathrm{CO}_{2}$ incubator set at $37^{\circ} \mathrm{C}$ for one day. On the following day, some of the pates were treated with siRNA (details of siRNA treatment described below). On the third day, some of the plates were treated with FG-

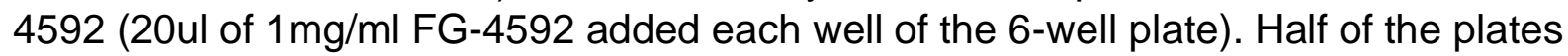
were incubated in normoxia $\left(21 \% \mathrm{O}_{2}, 5 \% \mathrm{CO}_{2}\right.$ at $\left.37^{\circ} \mathrm{C}\right)$ and rest half in hyperoxia $(75 \%$ $\mathrm{O}_{2}, 5 \% \mathrm{CO}_{2}$ at $37^{\circ} \mathrm{C}$ ) incubator for $24 \mathrm{~h}$. Following the incubation, RNA was extracted from the cells using TriReagent. Media was aspirated from the plates, following which cells were washed with $1 \mathrm{ml}$ of normal saline and $1 \mathrm{ml}$ of TriReagent was added to each plate. Plates were incubated at room temperature for $5 \mathrm{~min}$, following which cell lysate was transferred to $1.5 \mathrm{ml}$ tubes. Added $200 \mu \mathrm{l}$ of chloroform to each tube and vortexed for $1 \mathrm{~min}$. The samples were then incubated samples at room temperature for $10 \mathrm{~min}$ and subsequently centrifuged at $12000 \times \mathrm{g}$ for $15 \mathrm{~min}$ at $4^{\circ} \mathrm{C}$. The upper layer was transferred to fresh tubes containing $0.5 \mathrm{ml}$ of isopropanol, mixed and then incubated at room temperature for $10 \mathrm{~min}$. The samples were again centrifuged at $12000 \mathrm{x} \mathrm{g}$ for 10 min at $4^{\circ} \mathrm{C}$ and discarded the supernatants were discarded. The pellets were washed with $1 \mathrm{ml}$ of $75 \%$ ethanol followed by centrifugation at $7500 \times \mathrm{g}$ for $5 \mathrm{~min}$ at $4^{\circ} \mathrm{C}$. 
Discarded supernatant and air dried pellets. RNA was dissolved in $20 \mu \mathrm{l}$ of molecular biology grade water. RNA was converted to cDNA using Verso cDNA synthesis kit (Thermo Scientific) using anchored oligo-dT primers and following protocol provided with the kit.

\section{RT-qPCR}

Two microliters of cDNA was added to each well of 96 well plate, followed by addition of 1x Radiant ${ }^{T M}$ SYBR Green Lo-ROX (Alkali Scientific). Following qPCR primers were used

1) ARG1 Quantitech catalogue number QT00134288, 1x per reaction

2) HIF1 a Quantitech catalogue number QT01039542, 1x per reaction Self-designed primers

3) CPS1, Forward 5'- GTGAAGGTCTTGGGCACATC-3' Reverse 5'TTCCACTGCAAAACTGGGTG-3'

4) $\beta$-actin Forward 5'-TACTCTGTGTGGATCGGTGG-3' Reverse 5'TCGTACTCCTGCTTGCTGAT-3'

Quantitech primers were used at 1x concentration per well. Self-designed primers were used at a final concentration of $1 \mu \mathrm{l}$ of $10 \mu \mathrm{M}$ forward and $1 \mu \mathrm{l}$ of $10 \mu \mathrm{M}$ reverse, per well. Volume of each well was made to $20 \mu \mathrm{l}$ with molecular biology grade water. The qPCR program used was $50^{\circ} \mathrm{C}$ for $2 \mathrm{~min}, 95^{\circ} \mathrm{C}$ for $10 \mathrm{~min}, 40$ cycles at $95^{\circ} \mathrm{C}$ for $00: 15$ $\min , 60^{\circ} \mathrm{C}$ for $00: 20 \mathrm{~min}$, and $72^{\circ} \mathrm{C}$ for $00: 20 \mathrm{~min}$ with acquisition of fluorescence followed by a melting curve $95^{\circ} \mathrm{C}$ for $00: 15 \mathrm{~min}, 60^{\circ} \mathrm{C}$ for $1: 00 \mathrm{~min}, 95^{\circ} \mathrm{C}$ for 00:15 min.

\section{siRNA mediated knock-down}

Following ON-TARGETplus non-targeting pool from Dharmacon (Horizon Discovery):

1) ON-TARGETplus Mouse Hif1a (15251) siRNA - SMARTpool, catalogue number L-040638-00-0005

2) ON-TARGETplus Mouse Epas1 (13819) siRNA - SMARTpool, catalogue number L-040635-01-0005

3) ON-TARGETplus Non-targeting Pool, catalogue number D-001810-10-05

$5 \mathrm{nmol}$ of powdered siRNAs were dissolved in $100 \mu \mathrm{l}$ of water and mixed well with pipetting up and down couple of times. siRNA solutions were mildly vortexed before use. $25 \mu$ l of siRNA solution was mixed with $625 \mu$ l of low-serum Optimem media. In a separate tube, $75 \mu$ l of lipofectamine 2000 (Invitrogen) was mixed with $625 \mu \mathrm{l}$ of Optimem. Added siRNA/Optimem mixture to tube containing lipofectamine 2000/Optimem and incubated at room temperature for few mins. Media in the 6-well plates was changed with fresh low-serum Opimem, followed by the addition of $100 \mu l$ siRNA/lipofectamine/Optimem mixture per well. Plates were incubated for $5 \mathrm{~h}$ in $5 \% \mathrm{CO} 2$ incubator set at $37^{\circ} \mathrm{C}$. Following which media was changed to William's $\mathrm{E}$ media and further procedures were performed as described above. 


\section{Acknowledgements}

Grant Support: National Eye Institute (R01 EY024972 to JES; R01 EY026181 to BAA, R01 EY027083 to BAA, P30EY025585 to BAA); 5T32EY007157-19 grant to AG; Research to Prevent Blindness RPB1801 to JES. This work was supported in part by RPB Challenge grant and unrestricted grant from the Cleveland Eye Bank Foundation. We would like to thank Northern Ohio Alcohol Center for providing us freshly isolated hepatocytes, supported by grant P50AA024333 (PI Dr. Laura Nagy, Cleveland Clinic, Ohio).

Author Contributions: CS conceived, designed performed, analyzed, wrote the paper. AB performed experiments and data analysis, and edited the paper. AG performed experiments and edited the paper. VT performed experiments and analyzed data. $\mathrm{DH}$ performed experiments. BA conceived, designed and wrote the paper. HB conceived, designed and wrote the paper. JES conceived the experiments and wrote the paper.

Data availability All the mass spectrometry raw data are available online on metabolights, study id: MTBLS2221.

Study approval. All experimental procedures involving live animals were conducted in accordance with the guidelines of the NIH's Guide for the Care and Use of Laboratory Animals (National Academies Press, 2011) and approved by the Cleveland Clinic institutional animal care and use committee (IACUC, protocol 2016-1677). All animal experiments conformed to the rules and regulations of the Association of Research in Vision and Ophthalmology.

1. J. B. Spinelli et al., Metabolic recycling of ammonia via glutamate dehydrogenase supports breast cancer biomass. Science (New York, N.Y.) 358, 941-946 (2017).

2. Y. Wang et al., Coordinative metabolism of glutamine carbon and nitrogen in proliferating cancer cells under hypoxia. Nature Communications 10, 201 (2019).

3. J. Fang et al., Dihydro-orotate dehydrogenase is physically associated with the respiratory complex and its loss leads to mitochondrial dysfunction. Bioscience reports 33, e00021-e00021 (2013).

4. J. Sidney M. Morris, REGULATION OF ENZYMES OF THE UREA CYCLE AND ARGININE METABOLISM. 22, 87-105 (2002).

5. M. Takiguchi, M. Mori, Transcriptional regulation of genes for ornithine cycle enzymes. The Biochemical journal 312 ( Pt 3), 649-659 (1995).

6. C. Ulbright, P. J. Snodgrass, Coordinate induction of the urea cycle enzymes by glucagon and dexamethasone is accomplished by three different mechanisms. Arch Biochem Biophys 301, 237-243 (1993).

7. J. G. Okun et al., Molecular regulation of urea cycle function by the liver glucocorticoid receptor. Molecular Metabolism 4, 732-740 (2015).

8. Z. Chen, N. Tang, X. Wang, Y. Chen, The activity of the carbamoyl phosphate synthase 1 promoter in human liver-derived cells is dependent on hepatocyte nuclear factor 3-beta. J Cell Mol Med 21, 2036-2045 (2017). 
9. K. Nohara et al., Ammonia-lowering activities and carbamoyl phosphate synthetase 1 (Cps1) induction mechanism of a natural flavonoid. Nutrition \& metabolism 12, 23 (2015).

10. L. Li et al., p53 regulation of ammonia metabolism through urea cycle controls polyamine biosynthesis. Nature 567, 253-256 (2019).

11. C. Singh et al., Serine and 1-carbon metabolism are required for HIF-mediated protection against retinopathy of prematurity. JCl insight 4 (2019).

12. W. C. Hallows et al., Sirt3 promotes the urea cycle and fatty acid oxidation during dietary restriction. Mol Cell 41, 139-149 (2011).

13. T. Nakagawa, L. Guarente, Urea cycle regulation by mitochondrial sirtuin, SIRT5. Aging (Albany NY) 1, 578-581 (2009).

14. L. P. Paris et al., Global metabolomics reveals metabolic dysregulation in ischemic retinopathy. Metabolomics 12, 15 (2016). 